\title{
High Resolution TEM and Electron Diffraction Study of Graphene Layers
}

\author{
Y. J. Suh, S. Y. Park, and M. J. Kim \\ Dept. of Materials Science \& Engineering, University of Texas at Dallas, Richardson, TX 75080
}

Graphene, a single or few layers of carbon atoms placed in a hexagonal lattice, has been one of the more important materials for nanoelectronics due to its excellent electrical characteristics [1]. According to theoretical calculations, the properties strongly depend on the number of carbon atom layers and the presence of defects [2-3]. However the method to characterize graphene structure and layer stacking has not been well established. In order to identify the layer structure of graphene, diffraction patterns with different numbers of layers and/or different stacking sequences were obtained using JEMS simulation and compared with experimentally observed diffraction patterns [4]. For gathered information on an in-layer atomic structure, simulated HRTEM images of graphene are also compared with experimentally acquired images.

In order to produce a suspended graphene layer, a micromechanical cleavage method was adopted. Natural graphite and highly ordered pyrolytic graphite (HOPG) were stamped on to a meshed $\mathrm{Cu}$ grid pasted with an adhesive layer. HRTEM and diffraction pattern analyses were carried out in a JEOL 2100F TEM/STEM.

The intensity ratio of diffraction spots from $\{11-20\}$ to those from $\{1-100\}$ varied depending on the number of stacking layers. Simulation results revealed that graphenes with even number of stacking layers like $\mathrm{AB}, \mathrm{ABAB}$, etc showed a constant intensity ratio of 1.78 . On the other hand, graphenes with odd number of layers such as $\mathrm{A}, \mathrm{ABA}$, etc showed varying intensity ratios that increased with the number of layers. When there is only one layer, the intensity of the $\{1-100\}$ is stronger than that of the $\{11-20\}$. But as the number of layers increases, the intensity ratio increases; approaching the value of 1.78, the same as that for graphene with an even number of layers (Fig. 1). By analyzing experimentally observed diffraction patterns, we could distinguish single layer graphene from multilayer graphene as shown in Fig. 2. It should be noted that a three-layer graphene with a stacking sequence of $\mathrm{ABC},\{1-100\}$ diffraction spots disappear and double layer with AA stacking has the same intensity as that of a single layer. However, these stacking sequences are known to be unfavorable.

HRTEM images were obtained from a monolayer graphene sample, confirmed by the diffraction pattern as described above (Fig. 3). The center of the hexagon appears as one unit in the image, although six carbon atoms should be located at each corner of the hexagon. In order to confirm the experimental image, HRTEM images with different defocus values ranging from $-50 \mathrm{~nm}$ to $-10 \mathrm{~nm}$ were simulated. The simulated HRTEM image with the Scherzer defocus condition of $-45 \mathrm{~nm}$ showed tendencies similar to those of the experimental image [5].

[1] A. K. Geim and K. S. Novoselov, Nature Materials. 6 (2007) 183.

[2] J. R. Huang, J. Y. Lin, B. H. Chen, and M. H. Tsai, Phys. Stat. Sol.(b), 1 (2008) 245.

[3] Y.W. Son, M. L. Cohen, and S. G. Louie, Nature 444 (2006) 347.

[4] JEMS, P. Stadelmann,: http://cimewww.epfl.ch/people/stadelmann/jemsWebSite/jems.html

[5] This research was supported by the SWAN program funded by the GRC-NRI. 


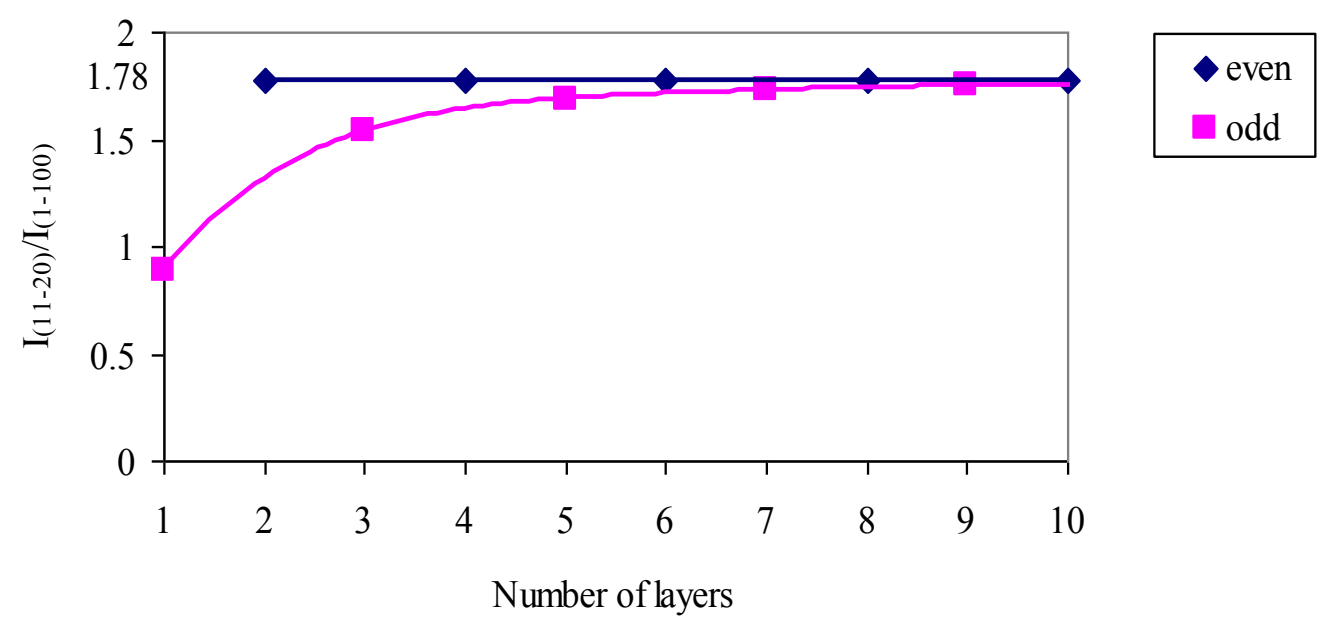

Fig. 1. Intensity ratio variation of $\{11-20\}$ and $\{1-100\}$ spots for graphenes depending on number of layers. Stacking sequence of graphene layers with $\mathrm{ABAB}$... was used for this calculation.
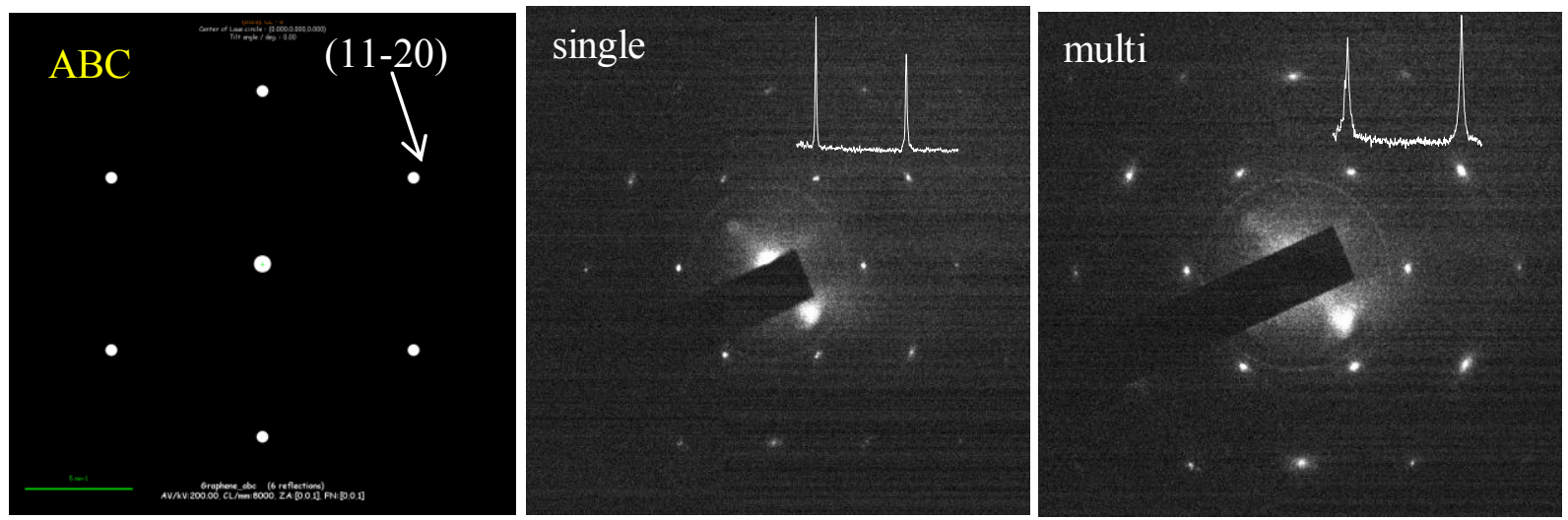

Fig. 2. A simulated diffraction pattern of graphene stacking $A B C$ (left), and diffraction patterns of monolayer and multi layer graphene (middle and right).
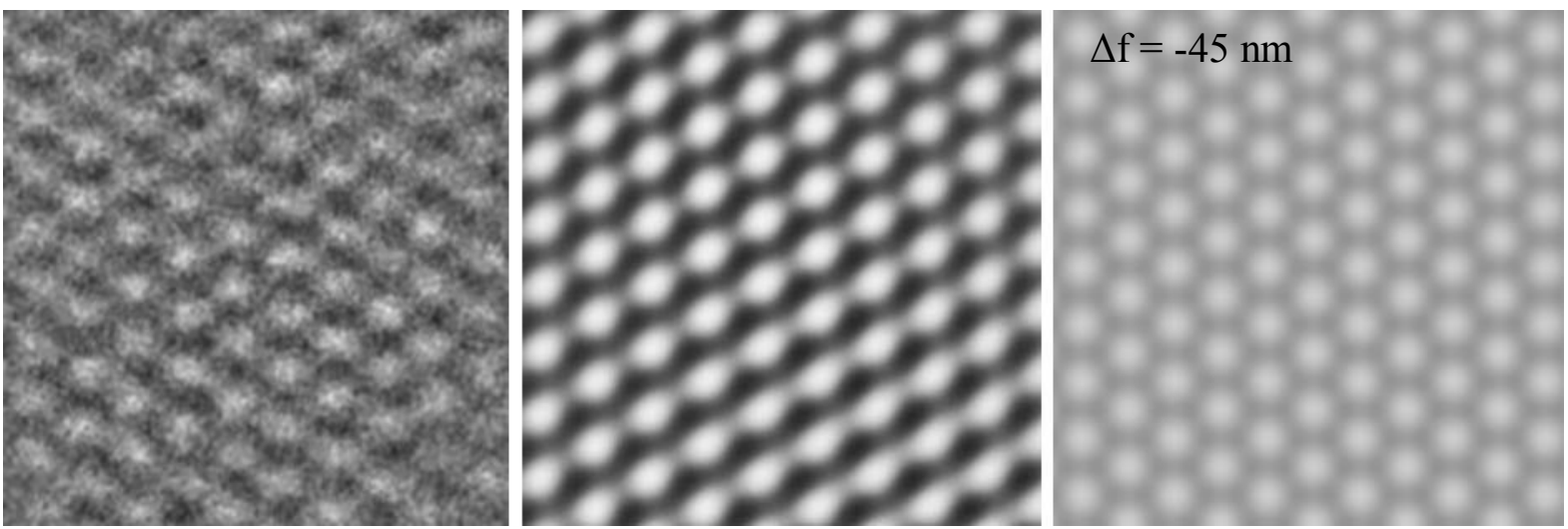

Fig. 3. HRTEM image, Inverse Fourier transformed image, and simulated HRTEM image of monolayer graphene are shown from left to right (Conditions: $\mathrm{C}_{\mathrm{s}}=0.5 \mathrm{~mm}$, and $\mathrm{V}=200 \mathrm{keV}$ ). 